\section{Accurate Solution of Proportional Navigation for Maneuvering Targets}

\author{
P. R MAHAPATRA \\ U. S. SHUKLA \\ Indian Institute of Science
}

An accurate solution is presented of the nonlinear differential equations describing motion under proportional navigation when the target is laterally maneuvering. A quasillnearization (QL) approach is used, followed by a perturbation technique to obtain closed-form solutions for trajectory parameters. Explicit expression for the pursuer lateral acceleration is derived and shown to contain contributions due to initial heading error and target maneuver, with a coupling between the two effects. The solution is shown to be a substantial and consistent generalization of our earlier accurate solution for nonmaneuvering targets and also of classical linear (CL) solutions for maneuvering targets The generalized QL solution presented here provides very accurate estimates of pursuer lateral acceleration over a much broader range of engagement geometries and target maneurers than presently available closed-form solutions.

Manuscript received February 18, 1988; revised May 23, 1988

IEEE Log No. 25819.

Authors' address: Department of Aerospace Engineering, Indian Institute of Science, Bangalore 560012, India.

$\overline{0018}-9251 / 89 / 0100-0081 \$ 1.00$ (C) 1989 IEEE

\section{Variables}

$\boldsymbol{A}$ Lateral acceleration (normal to velocity vector).

$\boldsymbol{N}$ Navigation constant.

$T$ Estimated time-to-go (range-to-go $\div$ initial closing velocity).

$\boldsymbol{V}$ Velocity.

$k \quad$ Normal turn rate of target $\left(A_{T} / V_{T}\right)$.

$k^{\prime} \quad$ Normalized turn rate of target $\left(k T_{i}\right)$.

$r \quad$ Range from pursuer to target.

$\boldsymbol{t}$ Time.

$t^{\prime} \quad$ Normalized time $\left(t / T_{i}\right)$.

$\phi \quad$ Angle between pursuer velocity vector and reference line.

$\Delta \phi$ Heading error relative to collision course.

$\boldsymbol{\theta}$ Angle of line of sight (LOS) relative to reference line.

P Angle between target velocity vector and reference line.

$\Delta \beta$ Incremental rotation of target velocity vector from its initial direction.

Subscripts

CL Classical linear.

$M$ Pursuer.

QL Quasilinear.

$\mathrm{T}$ Target.

$i \quad$ Initial values.

$f \quad$ Final value (at intercept).

0 Zeroth-order (initial) approximation.

c (for) Constant bearing course.

$r L$ Relative (velocity) along line of sight (LOS) .

riL Initial relative (velocity) along line of sight (LOS).

\section{INTRODUCTION}

Proportional navigation (PN) has been widely used as a guidance law for tactical appplications in the recent decades. It is very simple to implement on-board and is very efficient in a wide variety of geometrical situations. However, the analysis of PN-based guidance systems has been difficult, because the equations governing motion under the PN law are highly nonlinear. General analytical solutions to these equations have not been available even for the simple case of engagement against nonmaneuvering targets. Certain particular solutions have been reported earlier [1-3]. The specific, but practically less useful, case of true proportional navigation (TPN) has been solved by Guelman [4] for the nonmaneuvering target. The TPN solution has recently been generalized [5] in terms of the direction of the applied control force, but again only for rectilinear target motion. The more practical case of pure proportional navigation (PPN) has not been solved for a general value of the navigation constant, even for the nonmaneuvering target case.

The treatment of the PN problem for nonmaneuvering targets is of very little practical value 
since the overwhelming majority of PN application situations involve maneuvering targets. However, for maneuvering targets, the PN equations become highly intractable. No general solution to PN motion against maneuvering targets has been reported. Some qualitative treatment $[6,7]$ has been attempted, but such treatment has been confined to determining bounds on lateral accelerations rather than obtaining actual solutions for the pursuer trajectory parameters.

The only effective and general analytical method of analyzing PN motion has been hitherto based on linearization of the equations $[2,8,9]$. Because of the small-angle approximations necessary for linearization, the linearized solutions are valid only for near-tail-chase geometries and small target maneuvers. Further, the linear superposition of the individual contributions of lateral acceleration due to the target maneuver and the initial heading error does not remain valid for large heading errors.

We present a method of accurately solving the PPN equations in closed form when the target motion involves significant maneuver and initial heading errors. The method is based on a quasilinearization (QL) procedure [10] and may be viewed as a substantial extension of our recent paper [11] presenting similar solutions for nonmaneuvering targets. The explicit QL solutions presented here are shown to be a generalization of the classical linear (CL) solutions. This generalization results in an improvement in the estimation of pursuer lateral acceleration and enlarges the validity of the solutions to a much broader range of engagement geometries and maneuver levels than is possible with the CL approach. The method outlined here is capable of generating explicit solutions for all trajectory angles, as well as the time of flight and lateral acceleration. However, for space considerations, only the most important parameter, i.e., the pursuer lateral acceleration, is dealt with here.

\section{PROPORTIONAL NAVIGATION EQUATIONS FOR MANEUVERING TARGETS}

Consider the geometry of Fig. 1. The target is assumed to have a constant forward velocity $V_{T}$ and a constant lateral acceleration $A_{T}$. The differential equations describing the pursuer motion may be written as

$$
\dot{r}=V_{T} \cos (\theta-k t)-V_{M} \cos (\theta-\phi)
$$

and

$$
r \dot{\theta}=-V_{T} \sin (\theta-k t)+V_{M} \sin (\theta-\phi)
$$

where $k=A_{T} / V_{T}$ represents the normal turn rate of the target. Using the definition $\dot{\phi}=N \dot{\theta}$ of PPN in (1) and (2)

$$
\dot{r}=V_{T} \cos (\theta-k t)-V_{M} \cos (b \theta-c)
$$

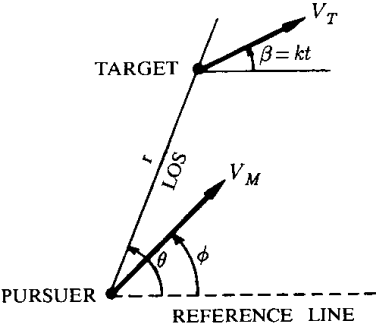

Fig. 1. Geometry of proportional navigation for maneuvering target.

and

$$
r \dot{\theta}=-V_{T} \sin (\theta-k t)+V_{M} \sin (b \theta-c)
$$

where

$$
\mathrm{b}=1-\mathrm{N} \quad \text { and } \quad c=\phi_{i}-N \theta_{i} .
$$

For the normal range of $\mathrm{PN}$ parameters, the range-to-go $\boldsymbol{r}$ is a monotonic function of time $\boldsymbol{t}$. Under the assumption of monotonicity, (3) and (4) can be rewritten with $\boldsymbol{r}$ as the independent variable:

$$
T_{i} \frac{d t^{\prime}}{d r}=\frac{1}{V_{T} \cos \left(\theta-k^{\prime} t^{\prime}\right)-V_{M} \cos (b \theta-c)} \triangleq d\left(\theta, k^{\bullet}\right)
$$

and

$$
r \stackrel{d \theta}{d r}=\frac{-V_{T} \sin \left(\theta-k^{\prime} t^{\prime}\right)+V_{M} \sin (b \theta-c)}{V_{T} \cos \left(\theta-k^{\prime} t^{\prime}\right)-V_{M} \cos (b \theta-c)} \triangleq g\left(\theta, k^{\prime}\right) .
$$

where the normalized time $t^{\prime}=t / T_{i}$ and $k^{\prime}=k T_{i}$, with $T_{i}=r_{i} / V_{r i L}$

\section{QUASILINEARIZATION OF PROPORTIONAL NAVIGATION EQUATIONS}

The coupled equations (6) and (7) are highly nonlinear and do not admit closed-form solution. We derive approximate equivalents of these equations using the QL technique. Expanding the right-hand side (RHS) of (7) and (6), respectively, in Taylor series in function space and truncating after the first derivative term,

$$
\begin{array}{r}
r \frac{d \theta_{1}}{d r}=g_{0}+h_{0}\left(\theta_{1}-\theta_{0}\right) \\
T_{i} \frac{d t_{1}^{\prime}}{d r}=d_{0}+e_{0}\left(\theta_{1}-\theta_{0}\right)
\end{array}
$$

where $d o \stackrel{\mathrm{A}}{=} d\left(\theta_{0}\right), e_{0} \stackrel{\mathrm{A}}{=} e\left(\theta_{0}\right) \stackrel{\mathrm{A}}{=} \partial d /\left.\partial \theta\right|_{\theta=\theta_{0}}$ and $g_{0} \stackrel{\mathrm{\Xi}}{=} g\left(\theta_{0}\right), h_{0} \stackrel{\mathbf{A}}{=} h\left(\theta_{0}\right) \stackrel{\mathrm{A}}{=} \partial g /\left.\partial \theta\right|_{\theta=\theta_{0}}$ and the subscript 0 denotes the initial approximation to the solution and 1 denotes the solution after the first iteration. Although more iterations can be performed to improve accuracy, in this work we stop at the first iteration in order to be able to obtain a closed-form solution. Thus $\theta_{1}$ is our final solution for the variable 8 . To simplify the 
notation, we replace $\theta_{1}$ with $\theta$ and $t_{1}$ with $t$ in the following treatment.

From definition, and after necessary simplification, $\mathrm{h}$ and $e$ are obtained as

$$
\begin{aligned}
& h \triangleq \frac{\partial g}{\partial \theta}=\frac{-b V_{M}^{2}-V_{T}^{2}+(1+b) V_{T} V_{M} \cos \left(N \theta+c-k^{\prime} t^{\prime}\right)}{\left[V_{T} \cos \left(\theta-k^{\prime} t^{\prime}\right)-V_{M} \cos (b \theta-c)\right]^{2}} \\
&+k^{\prime} \frac{d t^{\prime}}{d \theta} \frac{V_{T}^{2}-V_{T} V_{M} \cos \left(N \theta+c-k^{\prime} t^{\prime}\right)}{\left[V_{T} \cos \left(\theta-k^{\prime} t^{\prime}\right)-V_{M} \cos (b \theta-c)\right]^{2}} \\
& e \stackrel{\mathrm{A}}{=} \frac{d d}{\partial \theta}=-\frac{-V_{T} \sin \left(\theta-k^{\prime} t^{\prime}\right)+b V_{M} \sin (b \theta-c)}{\left[V_{T} \cos \left(\theta-k^{\prime} t^{\prime}\right)-V_{M} \cos (b \theta-c)\right]^{2}} \\
&+k^{\prime} \frac{d t^{\prime}}{d \theta} \frac{-V_{T} \sin \left(\theta-k^{\prime} t^{\prime}\right)}{\left[V_{T} \cos \left(\theta-k^{\prime} t^{\prime}\right)-V_{M} \cos (b \theta-c)\right]^{2}}
\end{aligned}
$$

Equations (8) and (9) with the definitions in (6), (7), (10), and (11) constitute the approximation to the nonlinear PN equations (6) and (7).

\section{PERTURBATION SOLUTION OF QUASILINEARIZATION EQUATIONS}

The QL equations (8) and (9) are not directly solvable in closed form. A perturbation series method is adopted here to obtain an approximate solution. The variables $\theta$ and $t^{\prime}$ are expressed as truncated perturbation series:

$$
\theta\left(r, k^{\prime}\right)=\theta_{N}(r)+k^{\prime} \theta_{M}(r)
$$

and

$$
t^{\prime}\left(r, k^{\prime}\right)=t_{N}^{\prime}(r)+k^{\prime} t_{M}^{\prime}(r)
$$

where subscript $N$ denotes the zeroth-order solution and $M$ denotes the first-order term. It is shown later that the zeroth-order term corresponds to the solution for nonmaneuvering target and the first-order term represents maneuver effects. For the series expansions (12) and (13) to be accurate, $\boldsymbol{k}^{\prime}$ must be small.

For the QL algorithm, an initial approximation to the solution is necessary. We choose the initial approximations

$$
\begin{aligned}
\theta_{N 0}(r) & =\theta_{i} ; \quad \theta_{M 0}(r)=0 \\
t_{N 0}^{\prime} & =t_{N 0} / T_{i} \\
& =\frac{\left(r_{i}-r\right) / V_{r i L}}{r_{i} / V_{r i L}}=\frac{r_{i}-r}{r_{i}} .
\end{aligned}
$$

Substituting (12) and (13) in (8) and (9) and using (14) in (10) and (11), and after simplification, retaining only the significant terms, we get, on equating the coefficients of $k^{\prime}$

$$
\begin{aligned}
& r \frac{d \theta_{N}}{d r}=G_{N 0}+H_{N 0}\left(\theta_{N}-\theta_{i}\right) \\
& r \frac{d \theta_{M}}{d r}=G_{M 0}+H_{N 0} \theta_{M}+H_{M 0}\left(\theta_{N}-\theta_{i}\right)
\end{aligned}
$$

and

$$
T_{i} \frac{d t^{\prime}}{d r}=T_{i} \frac{d t_{N}^{\prime}}{d r}=\frac{d t}{d r}=D_{N 0}+E_{N 0}\left(\theta_{N}-\theta_{i}\right)
$$

where

$$
\begin{aligned}
G_{N 0}= & \frac{-V_{T} \sin \theta_{i}+V_{M} \sin \left(b \theta_{i}-c\right)}{V_{t} \cos \theta_{i}-V_{M} \cos \left(b \theta_{i}-c\right)}=-\frac{r_{i} \dot{\theta}_{i}}{V_{r i L}} \\
G_{M 0}= & -\frac{V_{T}}{V_{r i L}} t_{N 0}^{\prime}\left(\cos \theta_{i}-G_{N 0} \sin \theta_{i}\right) \\
H_{N 0}= & \frac{-b V_{M}^{2}-V_{T}^{2}+(1+b) V_{T} V_{M} \cos \left(N \theta_{i}+c\right)}{\left[V_{T} \cos \theta_{i}-V_{M} \cos \left(b \theta_{i}-c\right)\right]^{2}} \\
H_{M 0}= & \frac{-2 H_{N 0} V_{T} t_{N 0}^{\prime} \sin \theta_{i}}{V_{T} \cos \theta_{i}-V_{M} \cos \left(b \theta_{i}-c\right)} \\
& +\frac{(1+b) V_{T} V_{M} t_{N 0}^{\prime} \sin \left(N \theta_{i}+c\right)}{\left[V_{T} \cos \theta_{i}-V_{M} \cos \left(b \theta_{i}-c\right)\right]^{2}} \\
D_{N 0}= & \frac{1}{V_{T} \cos \theta_{i}-V_{M} \cos \left(b \theta_{i}-c\right)}=-\frac{1}{V_{r i L}} \\
E_{N 0}= & -\frac{-V_{T} \sin \theta_{i}+b V_{M} \sin \left(b \theta_{i}-c\right)}{\left[V_{T} \cos \theta_{i}-V_{M} \cos \left(b \theta_{i}-c\right)\right]^{2}} .
\end{aligned}
$$

The zeroth-order equations (15) and (17) correspond to the nonmaneuvering case and have been solved in closed form in an earlier paper [11]. The solution is reproduced below.

$$
\begin{aligned}
\theta_{N}=\theta_{i}+\frac{G_{N 0}}{H_{N 0}}\left[\left(\frac{r}{r_{i}}\right)^{H_{N 0}}-1\right] \\
T_{i} t^{\prime}=t=r_{i}\left[\left(\frac{r}{r_{i}}-1\right)\left(D_{N 0}-\frac{E_{N 0} G_{N 0}}{H_{N 0}}\right)\right. \\
\left.+\frac{E_{N 0} G_{N 0}}{H_{N 0}\left(H_{N 0}+1\right)}\left(\left(\frac{r}{r_{i}}\right)^{H_{N 0}+1}-1\right)\right] .
\end{aligned}
$$

The solution to the first-order equation (16) for $\theta$ is obtained as

$\theta_{M}=\frac{V_{T}}{V_{r i L}} \frac{R-S}{H_{N 0}-1}\left[\left(1-\frac{r}{r_{i}}\right)+\frac{1}{H_{N 0}}\left(\left(\frac{r}{r_{i}}\right)^{H_{N 0}}-1\right)\right]$

where

$$
R=\cos \theta_{i}-G_{N 0} \sin \theta_{i}
$$

and

$$
S=-2 G_{N 0} \sin \theta_{i}+\frac{D_{N 0} G_{N 0}}{H_{N 0}} V_{M}(1+b) \sin \left(N \theta_{i}+\mathrm{c}\right) .
$$

Substituting the value of $\theta_{N}$ and $\theta_{M}$ from (24) and (26) in (12), we get

$$
\begin{aligned}
\theta= & \theta_{i}+\frac{G_{N 0}}{H_{N 0}}\left[\left(\frac{r}{r_{i}}\right)^{H_{N 0}}-1\right]+\frac{r_{i}}{V_{r i L}^{2}} A_{T} \frac{(R-S)}{H_{N 0}-1} \\
& \times\left[\left(1-\frac{r}{r_{i}}\right)+\frac{1}{H_{N 0}}\left(\left(\frac{r}{r_{i}}\right)^{H_{N 0}}-1\right)\right] .
\end{aligned}
$$


Now the pursuer acceleration $A_{M}$ is given as

$$
A_{M}=V_{M} N(d \theta / d t)=V_{M} N(d \theta / d r) /(d t / d r) .
$$

Differentiating (28) and substituting for $G_{N 0}$ from (18)

$$
\begin{aligned}
& \underset{d r}{d A}=-\frac{\dot{\theta}_{i}}{V_{r i L}}\left(\frac{r}{r_{i}}\right)^{H_{N 0}-1} \\
& +\frac{A_{T}}{V_{r i L}^{2}} \frac{(R-S)}{H_{N 0}-1}\left[\left(\frac{r}{r_{i}}\right)^{H_{N 0}-1}-1\right]
\end{aligned}
$$

and, similarly, differentiating (24)

$$
\frac{d t}{d r}=D_{N 0}+\frac{E_{N 0} G_{N 0}}{H_{N 0}}\left[\left(\frac{r}{r_{i}}\right)^{H_{N 0}}-1\right]=-\frac{1}{V_{r L}} .
$$

When there is no initial heading error, i.e., $\dot{\theta}_{i}=0$, (30) and (31) reduce, respectively, to

$$
\frac{d \theta}{\mathrm{d} \mathrm{r}}=\frac{\mathrm{A}_{\mathrm{T}}}{V_{r i L}^{2}} \frac{\cos \theta_{i}}{H_{N 0}-1}\left[\left(\frac{r}{r_{i}}\right)^{H_{N 0}-1}-1\right]
$$

and

$$
\frac{d t}{d r}=D_{N 0}=-\frac{1}{V_{r i L}}
$$

Substituting (30) and (31) in (29), we obtain

$$
\begin{aligned}
A_{M}= & N V_{M} \dot{\theta}_{i} \frac{V_{r L}}{V_{r i L}}\left(\frac{r}{r_{i}}\right)^{H_{N 0}-1} \\
& +\frac{N V_{M} A_{T} V_{r L}}{V_{r i L}^{2}} \frac{R-S}{H_{N 0}-1}\left[1-\left(\frac{r}{r_{i}}\right)^{H_{N 0}-1}\right] .
\end{aligned}
$$

In the absence of initial heading error $\left(\dot{\theta}_{i}=0\right)$, (34) reduces to

$$
A_{M}=\frac{N V_{M} A_{T}}{V_{r i L}} \frac{\cos \theta_{i}}{H_{N 0}-1}\left[1-\left(\frac{r}{r_{i}}\right)^{H_{N 0}-1}\right] .
$$

Equation (34) is the final general QL solution for the pursuer lateral acceleration $A_{M}$ against a target maneuvering with a lateral acceleration $A_{T}$.

\section{COMPARISON WITH UNEAR SOLUTION}

In this section we show that the solution represented by (34) and (35) for a maneuvering target is a true generalization of the more restrictive solutions available earlier. Since the formulation of this paper is essentially a perturbation over the QL solution for nonmaneuvering targets [11], it is easy to see from (34) that $A_{T}=0$ (i.e., no maneuver) results in the second term vanishing, leaving only the first term which is the nonmaneuvering solution in [11].

To show the consistency of the general solution (34) with the CL formulations, we first obtain a compatible form of CL solution. Starting from (1) and
(2) and using small-angle approximations on $\mathrm{A} \$, \Delta \beta$, and $\theta$, we obtain [9]

$$
\ddot{\theta}\left(t_{f}-t\right)-2 \dot{\theta}=-\frac{V_{M}}{V_{r i L}} \cos \phi_{c} \phi+\frac{V_{T}}{V_{r i L}} \cos p i p
$$

where $\beta=\beta_{i}+\Delta \beta$ is equivalent to $k t$ in (1) and (2) and $\phi=\phi_{c}+\Delta \phi$. The solution of (36) is

$$
\begin{aligned}
\dot{\theta}= & -\frac{V_{T} \dot{\beta} \cos \beta_{i}}{V_{r i L}\left(2-N^{\prime}\right)}\left[1-\left(\frac{t_{f}-t}{t_{f}}\right)^{N^{\prime}-2}\right] \\
& +\dot{\theta}_{i}\left(\frac{t_{f}-t}{t_{f}}\right)^{N^{\prime}-2}
\end{aligned}
$$

where $N^{\prime}=N\left(V_{M} / V_{r i L}\right) \cos \phi_{c}$ is the effective navigation constant. Since

$$
\dot{\theta}_{i}=-\left(V_{M} \cos \phi_{c} / r_{i}\right) \Delta \phi_{i}
$$

for small angles, using (29)

$$
\begin{aligned}
& A_{M}=-\frac{V_{M}}{t_{f}} N^{\prime}\left(\frac{t_{f}-t}{t_{f}}\right)^{N^{\prime}-2} \Delta \phi_{i} \\
& +\begin{array}{c}
\cos \beta_{i} \\
\cos \beta_{i} \\
\cos \phi_{c} \\
\cos N^{\prime}-2 \\
N^{\prime}-2
\end{array}\left[1-\left(\frac{t_{f}-t}{t_{f}}\right)^{N^{\prime}-2}\right] A_{T} \\
& =-\frac{V_{M} V_{r i L}}{r_{i}} N^{\prime}\left(\frac{r}{r_{i}}\right)^{N^{\prime}-2} \Delta \phi_{i} \\
& +\frac{N^{\prime}}{N^{\prime}-2} \frac{1}{\cos \phi_{c}}\left[1-\left(\frac{r}{r_{i}}\right)^{N^{\prime}-2}\right] A_{T}
\end{aligned}
$$

where $\beta_{i}$ has been assumed zero without loss of generality. The CL solution (39) has a form similar to the expression by Brainin and McGhee [S] when the bias term is zero.

To compare the QL solution with the CL solution, we first recast (32) in terms of $N^{\prime}$ and $\Delta \phi_{i}$, using (38), as

$$
\begin{aligned}
A_{M}= & -\frac{V_{M} V_{r L}}{r_{i}} N^{\prime}\left(\frac{r}{r_{i}}\right)^{H_{N 0}-1} \Delta \phi_{i} \\
& +\frac{N^{\prime}}{H_{N 0}-1} \frac{R-S}{\cos \phi_{c}} \frac{V_{r L}}{V_{r i L}}\left[1-\left(\frac{r}{r_{i}}\right)^{H_{N 0}-1}\right] A_{T}
\end{aligned}
$$

The general solution (40) has two distinct parts, the first corresponding to a nonmaneuvering target and the second providing the contribution due to target maneuver. However, unlike the CL solution (39), where the effects of initial heading error and target maneuver are distinct and uncoupled, here the second term in (40) also contains cross-coupling between $A_{T}$ and $\Delta \phi_{i}$. 


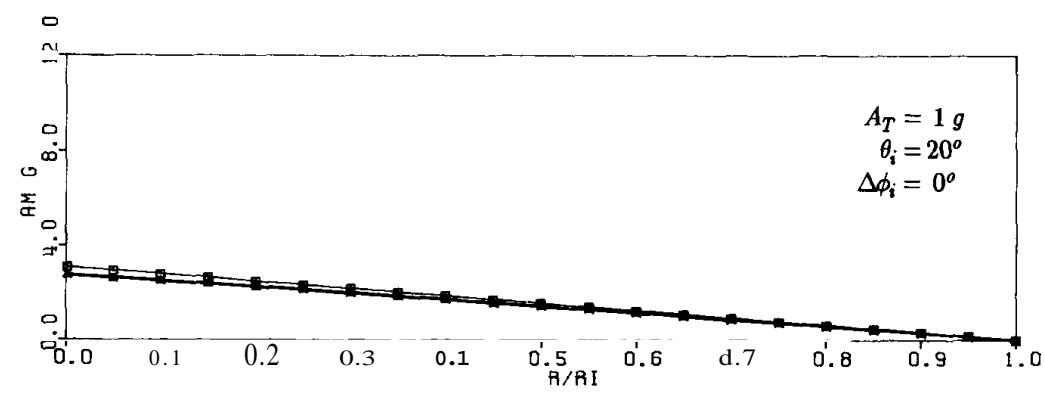

○

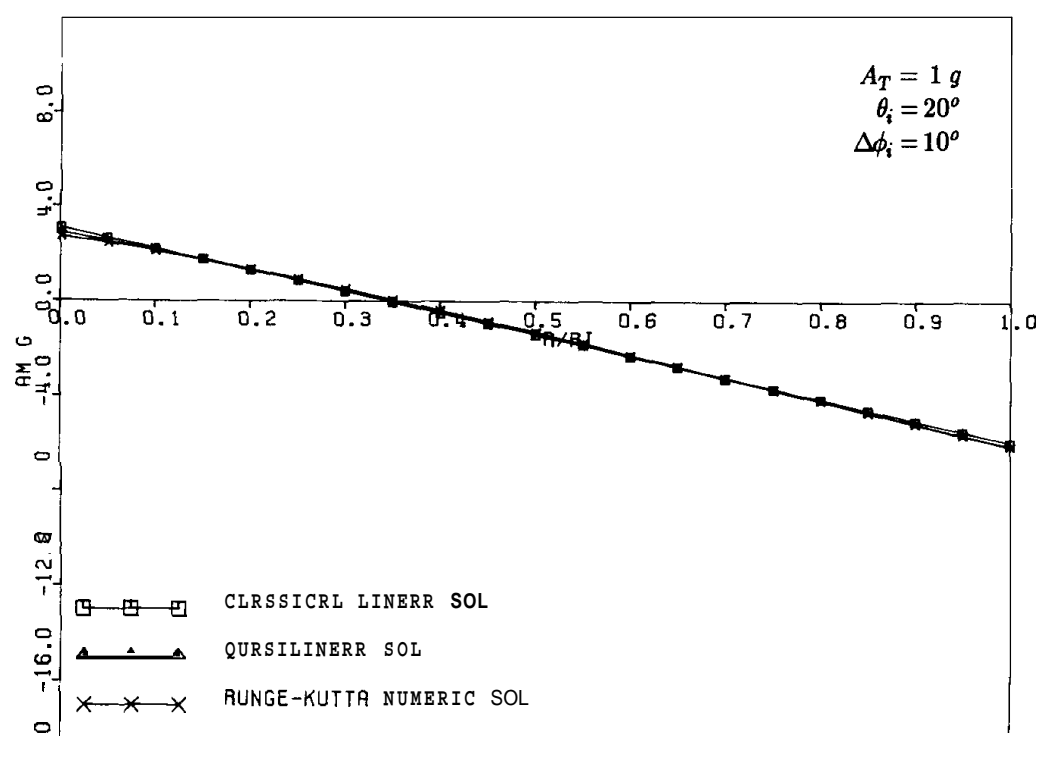

(a)

Fig. 2 Pursuer lateral acceleration (in gs) as function of normalized range. Target velocity is $300 \mathrm{~m} / \mathrm{s}$ and pursuer velocity is $900 \mathrm{~m} / \mathrm{s}$. Initial range is $\mathbf{5 m}$ and effective navigation constant is 3 for all plots. Target is assumed to have constant lateral acceleration $A_{T}$ of value specified in each plot.

For a near-tailchase situation, as assumed for the CL solution, $\theta_{i}$ and $\Delta \phi_{i}$ are small and (40) reduces to

$$
\begin{aligned}
A_{M} \cong & -\frac{V_{M} V_{r i L}}{r_{i}} N^{\prime}\left(\frac{r}{r_{i}}\right)^{H_{N 0}-1} \Delta \phi_{i} \\
& +\frac{N^{\prime}}{H_{N 0}-1} \frac{1}{\cos \phi_{c}}\left[1-\left(\frac{r}{r_{i}}\right)^{H_{N 0}-1}\right] A_{T}
\end{aligned}
$$

It is readily seen that the coupling between $A_{T}$ and $\Delta \phi_{i}$ has disappeared in (41) because of the small-angle approximation.

The QL result (41) is identical to the CL result (39) if $H_{N 0}$ in the former is replaced by $N^{\prime}-1$ in the latter. Indeed, $H_{N O}$ is a refinement of the classical effective navigation constant $N^{\prime}$ as has been established in [11]. The QL solution for a maneuvering target derived in this paper is thus a consistent generalization of the $\mathrm{CL}$ treatment.

\section{RESULTS AND DISCUSSION}

Fig. 2(a), (b), and (c) show plots of the lateral acceleration $A_{M}$ of the pursuer engaging a maneuvering target. The QL estimate of $\boldsymbol{A}_{\boldsymbol{M}}$ is obtained from (34) and (35) for pursuit with and without initial heading error, respectively. The results derived from CL formulation (39) are shown alongside for comparison. Further, for ascertaining the absolute accuracy of the results, the "exact" estimates of $A_{M}$ are also plotted in the figures. The exact estimates are obtained by an accurate numerical solution of the original nonlinear equations (3) and (4) for $\dot{\theta}$ using a $4 / 5$ th-order Runge-Kutta-Fehlberg algorithm [12] and substituting this $\dot{\boldsymbol{\theta}}$ in (29) to obtain $\boldsymbol{A}_{M}$.

In Fig. 2, a most commonly used effective navigation constant N'value of 3 is considered. Fig. 2(a) depicts results for small angles of the geometry, representing a near-tailchase pursuit, and also relatively low target maneuver. For this case, all the 

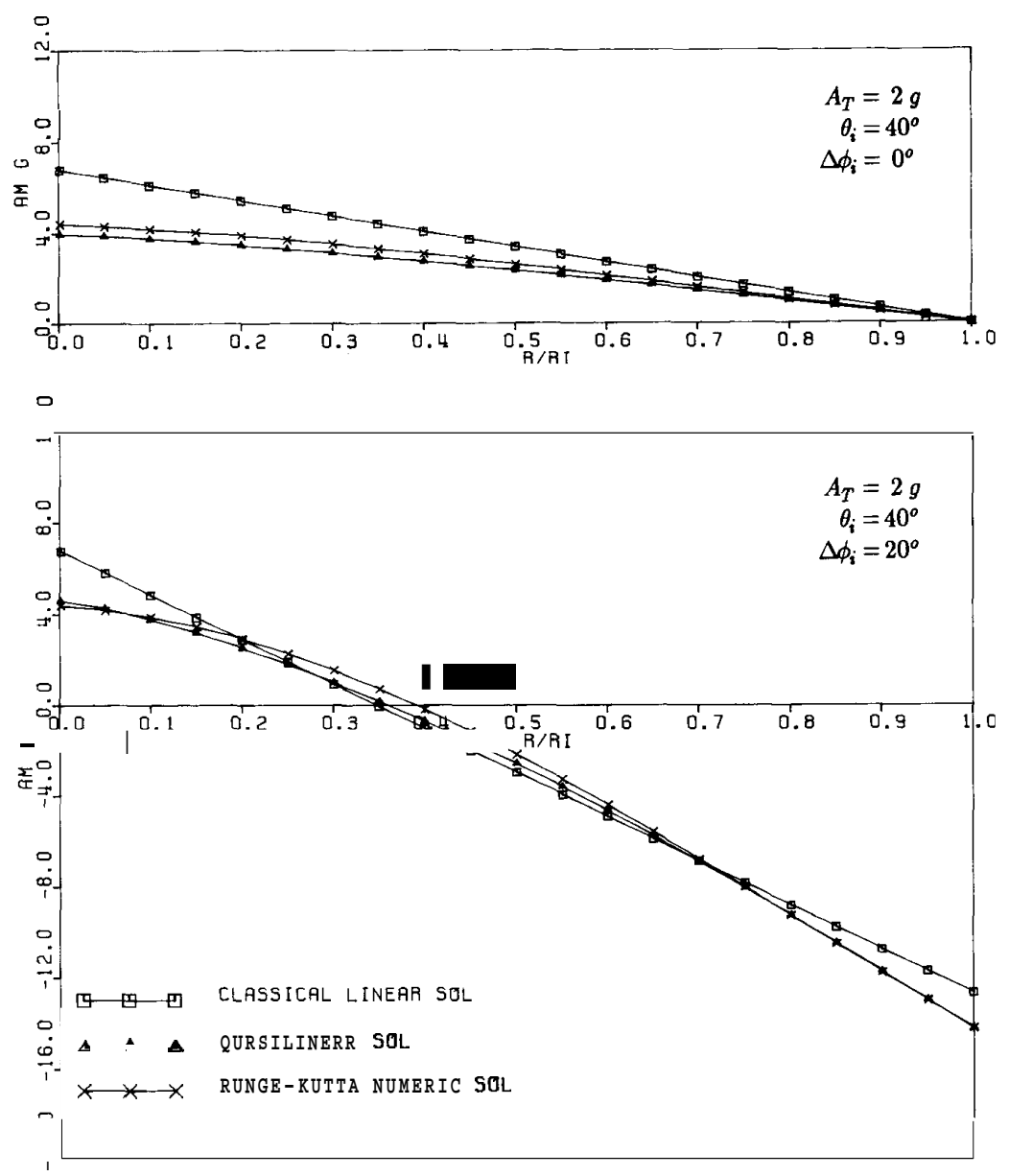

(b)

Fig. 2 Continued

three estimates (QL, linear, and "exact") of $A_{M}$ are found to be close. This is as would have been expected, since linear solutions for maneuvering targets are also based on such assumptions. However, even for this situation, the QL result shows a much closer coincidence with the exact results than the linear treatment.

In Figs. 2@) and (c), the angles and the target maneuver levels are progressively increased to high values. This represents a highly generalized pursuit scenario, with high target maneuvers and engagement geometries that are far from tail-chase and collision-course situations. The linear solutions currently available are not expected to be valid under such conditions, and they indeed are not, as seen from Figs. 2@) and (c). However, the QL technique of this paper continues to yield much more accurate results even under such general conditions.

While the foregoing results demonstrate the validity of the closed-form solutions developed earlier in the paper, some discussions on the general convergence properties of the $\mathrm{PN}$ equations are in order. We recall that the original PN equations (1) and (2) for maneuvering targets were recast as (6) and (7) which were solved in closed form through a two-step approximation. The first step was to assume a truncated Taylor series expansion of the RHS of the two equations around the initial launch angle $\theta_{i}$, and the second step involved a further approximation through a truncated perturbation series. In either case, only terms up to the first order have been retained. It is possible to comment on the convergence properties of the approximations.

From the plots in Fig. 2, it is seen that bulk of the error existing between the CL solution and the exact solution (as obtained numerically) has been accounted for by incorporating the generalization resulting from the inclusion of the linear terms in the expansions. Similar exercises have been carried out for a wide variety of combinations of pursuit parameters and it has been consistently observed that the first-order term helps the solution converge toward the exact value. 

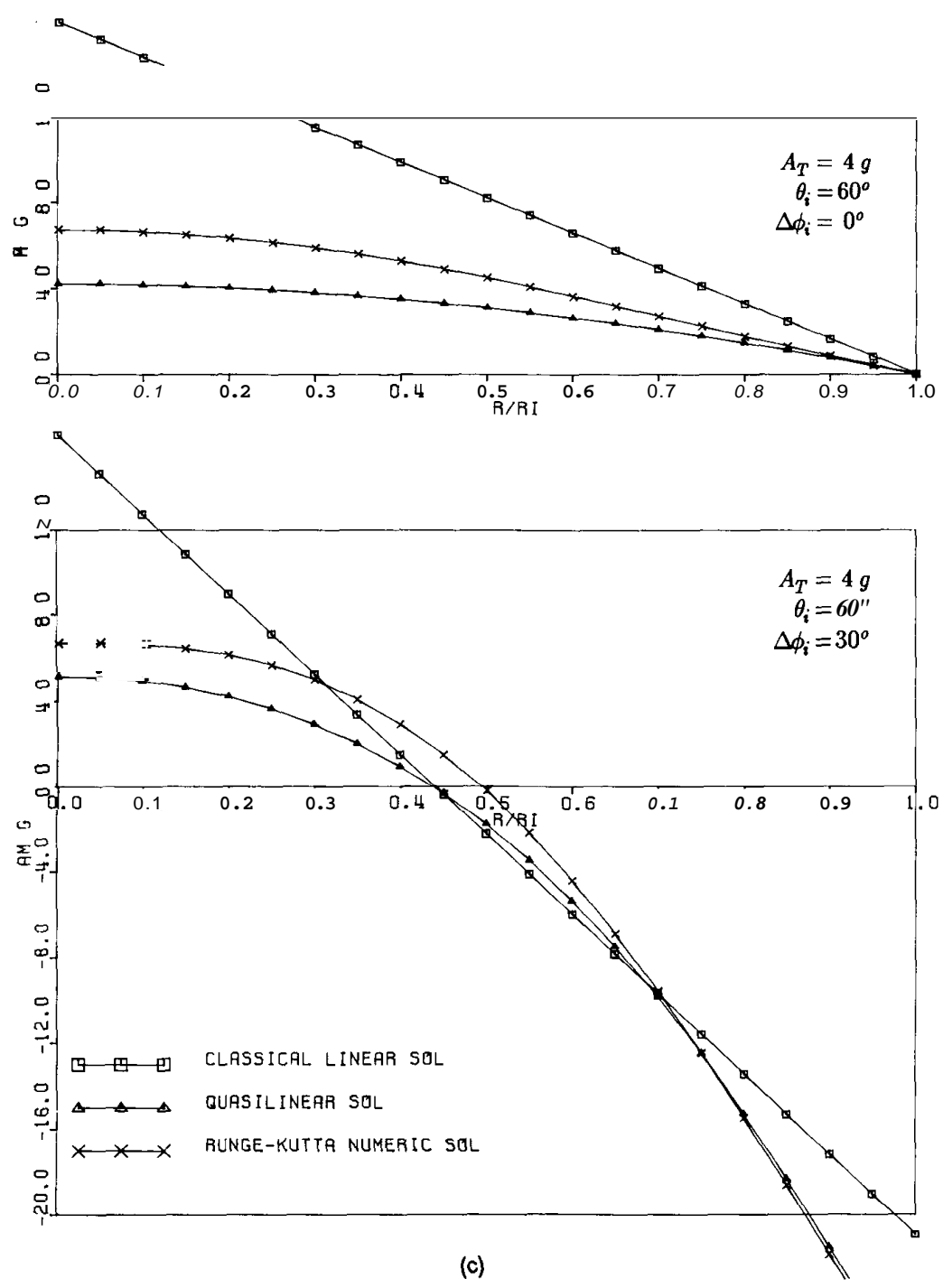

Fig. 2. Continued

To investigate the convergence behavior of the Taylor expansions, we considered one more term, i.e., the second-order term, in addition to the terms given in (8) and (9). This resulted in an expression for the line of sight (LOS) angle which is too involved to present here, even after neglecting the coupling terms between the maneuvering and nonmaneuvering parts of the solution. However, computations based on this extension show that inclusion of a second-order term in the Taylor expansions takes the solution closer to the exact solutions.

The convergence properties of perturbation series solutions have been well studied. The perturbation involved in (12) and (13) is clearly a regular perturbation problem, in which the zeroth-order solution is indeed the solution of the unperturbed problem, i.e., the nonmaneuvering case. The first-order solution determines the leading behavior of the final solution, as seen from the plots in Fig. 2 In such a case the remaining terms would only give small corrections [13].

No claims are made here regarding the optimality of the approximations used in this paper with regard to rate of convergence. In fact, the problem is too intractable to take a formal optimal approach to the convergence problem. It is, however, possible to try out alternative approaches, such as recasting the equations in different variable before expanding in Taylor series, trying other series than Taylor's, interchanging the sequence of application of the perturbation and Taylor series expansions, etc. Some of these have been tried out by the authors. However, the experience has been that generally the resulting approximate equations are not solvable 
in closed form, yielding explicit expressions for the trajectory parameters. In certain cases, it is possible to get explicit analytical solutions but they differ in structural form from the established CL solutions. The approach taken in this paper has resulted in the most general form of a hierarchy of solutions which can be progressively reduced to the nonmaneuvering case and further on to the CL solutions under suitable assumptions.

\section{CONCLUDING REMARKS}

$\boldsymbol{A n}$ attempt has been made to solve the difficult problem of obtaining the trajectory parameters of a projectile pursuing a maneuvering target under the pure proportional guidance strategy. Currently, only linear solutions of the nonlinear equations governing PN motion are available, the validity of which is restricted to low target maneuvers and to nearly tail-chase and collision-course pursuits. A QL approach has been followed in this paper to obtain closed-form solutions for the pursuer lateral acceleration which are found to yield accurate results for a wide range of engagement geometries and target maneuver levels.

It is shown analytically that the closed-form solution for the maneuvering target is a generalization of our recent quasilinear solution for nonmaneuvering targets, and also that the solutions reduce to the CL form for maneuvering targets, under the small-angle approximations employed for the linear solutions. The solution presented in this paper may thus be viewed as the highest member of a hierarchy of solutions of increasing generalization in the PN context.

The validity of the formulation, and also the fact of its generalization over the linear approach, is also demonstrated from the actual results derived from the formulation, using the exact numerical solution of the original PN equations as the standard. Even under conditions where the linear solutions are also valid, the generalized quasilinear solution of this paper shows a distinct improvement in lateral acceleration estimates; when the conditions become more severe and the linear solution ceases to be applicable, the quasilinear solution still continues to follow the true solution quite faithfully.

The treatment in this paper has been based on kinematic considerations only. The inclusion of dynamics would certainly be a useful step. However, the consideration of any worthwhile dynamics would render the problem rather intractable. It must be remembered that the kinematic problem itself is highly complex for PN motion against maneuvering targets, and no general closed-form solutions have been reported. In this context, the solution presented in this paper constitutes a definite contribution to the understanding of a very widely used guidance system such as proportional navigation. Furthermore, the approach utilized in this paper, and also the preceding one on nonmaneuvering targets, offers the potential for analytically handling problems of increasing complexity in the analysis of guidance and navigation systems.

\section{REFERENCES}

[1] Locke, A. S. (1955) Guidance.

Princeton, NJ.: Van Nostrand, 1955.

[2] Jerger, J. J. (1960) Systems Preliminary Design. Princeton, NJ.: Van Nostrand, 1960.

[3] Murtaugh, S. A. and Criel, H. E. (1966) Fundamentals of proportional navigation. IEEE Spectrum, 3 (Dec. 1966), 75-85.

[4] Guelman, M. (1976) The closed form solution of true proportional navigation. IEEE Transactions on Aerospace and Electronic Systems, AES-12 (July 1976), 472-482.

[5] Yang, C., Yeh, E, and Chen, J. (1987) The closed form solution of generalized proportional navigation. Journal of Guidance, Control and Dynamics, 10 (Mar.-Apr. 1987), 216-218.

[6] Guelman, M. (1972)

Proportional navigation with a maneuvering target. IEEE Transactions on Aerospace and Electrmic Systems, AES-8 (May 1972), 364-371.

[7] Guelman, M. (1973)

Missile acceleration in proportional navigation. IEEE Transactions on Aerospace and Electronic Systems, AES-9 (May 1973), 462-463.

[8] Brainin, S. M., and McGhee, R. B. (1968) Optimal biased proportional navigation. IEEE Transactions on Automatic Control, AC-13 (Aug. 1968), $440-442$

[9] Shinar, J. (1973)

Homing of a rolling missile against a maneuvering target. Israel Journal of Technology, 11 (1973), 117-130.

[10] Bellman, R., and Kalaba, R. (1965) Quasilinearization and Nonlinear Boundary Value Problems.

New York American Elsevier, 1965.

[11] Shukla, U. S., and Mahapatra, P. R. (1988) A generalized linear solution of proportional navigation. IEEE Transactions on Aerospace and Electronic Systems, AES-24, 3 (May 1988), 231-238.

[12] Forsythe, G. E., Malcolm, M. A., and Moler, C. B. (1977) Computer Methods for Mathematical Computations. Englewood Cliffs, NJ.: Prentice-Hall, 1977.

[13] Bender, C. M., and Orszag, S. A. (1978) Advanced Mathematical Methods for Scientists and Engineers.

New York McGraw-Hill, 1978. 

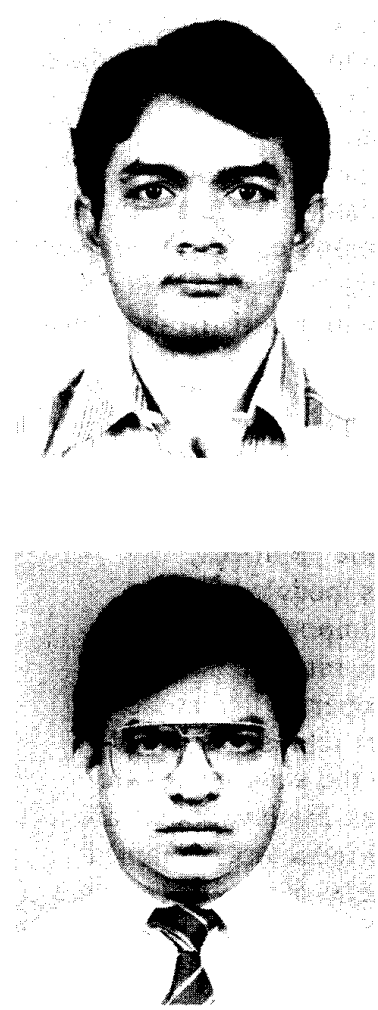

Pravas R. Mahapatra received the B.Sc. (Engg) from Regional Engineering College at Rourkela, and the M.E. and Ph.D. degrees from the Indian Institute of Science at Bangalore, India.

Dr. Mahapatra has been teaching at the Department of Aerospace Engineering in the Indian Institute of Science since 1970, where he is currently an Associate Professor. He has a broad area of active interest within the field of aerospace and electronic systems. This includes radar systems, navigational aids, and navigation theory and flight safety aspects with particular reference to weather phenomena and air traffic control. Dr. Mahapatra is a member of the U.S. Institute of Navigation.

Uday S. Shukla was born in $\mathbf{1 9 5 1}$ in Mathura (U.P.), India. He received the Bachelor's degree in electrical communication engineering in 1973 from the Indian Institute of Science, Bangalore, India.

Since 1973, he has been working with Hindustan Aeronautics Limited, Bangalore. His areas of interest lie in the broad field of avionic systems, and navigation theory and software. Currently, Mr. Shukla is an external registrant for the Ph.D. program at the Indian Institute of Science. 